like headache, nervousness and depression and, the low protection, with irritative eye symptoms, headache, nervousness and depression. The use of modern application technologies was negatively associated with skin irritation. Low levels of PPE use, lifetime exposure and lack of safe environments with appropriate technologies, involves higher levels of cumulative exposure, resulting in greater negative impact on their health.

\section{P2-173 CHRONIC DISEASES: STUDYING AND UNDERSTANDING OUTCOME USING ROUTINE DATA: CHRONIC KIDNEY DISEASE (CKD), AN EXAMPLE}

doi:10.1136/jech.2011.142976j.8

A Marks, ${ }^{*}$ N Fluck, G Prescott, A MacLeod, W C S Smith, C Black. Aberdeen Applied Renal Research Collaboration (ARRC), University of Aberdeen \& NHS Grampian, Aberdeen, Scotland, UK

Internationally, chronic disease represents a major healthcare challenge for the $21^{\text {st }}$ century. Prognostic tools that streamline and target care have been developed for cardiovascular disease. This study illustrates how routine data can be used to develop tools for other chronic diseases; using chronic kidney disease (a precursor of renal replacement therapy (RRT: dialysis or transplantation) requirement) as a model. Routine clinical data-serum creatinine (a measure of kidney function), RRT initiation and death registration were used to identify a CKD cohort, and follow them over 6 years. Mortality was compared to the general population. 3426 persons were identified with CKD (median age 79 years, $56 \%$ female). RRT initiation rates decreased with age from 14.3 to 0.7 per 100 personyears among those aged $15-25$ and $75-85$ years at baseline respectively (absolute numbers 6 and 34). Mortality rates increased with age from 1.9 to 33.8 per 100 person-years for those aged $15-45$ and over 85 years at baseline-a 19 and 2 fold increase in mortality risk compared to the general population respectively ( 2 and 17 excess deaths per 100 person-years). CKD has been labelled a public health concern, and provides a typical pattern for chronic disease. Personal risk is low for the majority, but they represent a high societal cost; whereas those with high personal risk are few, with lower societal cost. Exploitation of routinely collected data are an efficient way of following up health outcome, and informing the development of prognostic tools for a chronic disease cohort.

\section{P2-174 SURVIVAL ANALYSIS OF CYSTIC FIBROSIS PATIENTS IN A REFERENCE CENTRE IN RIO DE JANEIRO, BRAZIL}

doi:10.1136/jech.2011.142976j.9

${ }^{1} \mathrm{~L}$ S Higa, ${ }^{1,2} \mathrm{~V}$ Andreozzi, ${ }^{1,3} \mathrm{P}$ H Cabello, ${ }^{1} \mathrm{M}$ V M Peixoto. ${ }^{1} I F F$, FIOCRUZ, Rio de Janeiro, Rio de Janeiro, Brazil; ${ }^{2}$ CEAUL, Lisboa, Portugal; ${ }^{3}$ IOC, FIOCRUZ, Rio de Janeiro, Rio de Janeiro, Brazil

Cystic Fibrosis (CF) is a rare genetic disease, of autosomal recessive transmission, with multiple organ involvement, a progressive course and is potentially lethal. We studied the factors associated with the reduced survival. In an open cohort of cases diagnosed between 01 January 1990 and 10 October 2009 in a CF reference centre in Rio de Janeiro, we analysed survival and risk factors associated with survival. Information on patients included that on CF diagnostic criteria follow-up and outcome. The model included variables on gender, genotype, number of involved organs, nutritional state, bacterial colonisation, enzyme replacement and calendar-time of diagnosis. Survival was estimated by Kaplan-Meier (KM) method and covariates examined by log-rank tests. HRs were estimated by a Cox model and evaluated by the likelihood ratio, deviance and residual analysis. The majority of the population $(n=177)$ was female $(56 \%)$ and the median age at diagnosis was 14 months. The median survival was 19 years. After diagnosis, $81 \%$ survived up to 5 years, $70 \%$ up to 10 and $61 \%$ up to 14.5 . The model explained $19.9 \%$ of the effects and included six covariates. HRs were 10.30 (2.41-43.97) for isolated pseudomonas colonisation, 4.50 (0.93-1.85) for Staphylococcus aureus, 3.38 (0.92-1.32) for other bacteria, 1.95 (0.96-3.96) for gender, 1.94 (0.94-3.98) for nutritional state and 4.34 (1.50-12.52) for decade of diagnosis. Risk factors obtained at diagnosis were associated with prognosis suggesting that interventions may reduce morbidity by nutritional improvement and pseudomonas eradication.

\section{P2-175 MATERNAL SMOKING AND HEIGHT IN THE ADOLESCENT OFFSPRING. THE 1993 PELOTAS BIRTH COHORT}

doi:10.1136/jech.2011.142976j.10

1J Martínez-Mesa, * ${ }^{1}$ A M B Menezes, ${ }^{2} D$ A González, 'D P Gigante, 'B L Horta, ${ }^{1} \mathrm{C}$ L P Araújo, ${ }^{1}$ P C Hallal, ${ }^{1} \mathrm{H}$ Gonçalves. ${ }^{1}$ Posgraduated Program of Epidemiology, Pelotas, Rio Grande do Sul, Brazil; ${ }^{2}$ Federal University of Santa Catarina, Florianópolis, Santa Catarina, Brazil

Background Maternal smoking has been indicated as a risk factor for several health outcomes in the offspring.

Objective The aim of this work was to describe the association between maternal smoking during prenatal and postnatal periods and the offspring's height during adolescence.

Methods The 1993 Pelotas birth cohort (Southern Brazil) has primary data from birth to adolescence with several follow-ups and it comprised 5249 live-born newborns. The follow-up rates at 11 and 15 years old were, respectively: $87.5 \%$ and $85.7 \%$. The variable maternal smoking was categorised as: never smoker, only prenatal smoker, only postnatal smoker (during first year of life) and always smoker. Height was used as height for age z-score at 11 and 15 years using WHO curves. The confounding variables taken into account were: maternal height, maternal age, maternal schooling, paternal smoking, family income, sex, skin colour, Tanneŕs stage and adolescent smoking.

Results After adjustment for potential confounders in a multiple linear regression model, maternal smoking showed a significant and negative association ( $\beta$ values) with height for age $z$-score: a) at 11 years old [never smoker as the reference]: only prenatal smoker $(-0.47)$, only postnatal smoker $(-0.12)$, always smoker $(-0.30)$ $\mathrm{p}<0.001$; b) at 15 year old: only prenatal smoker $(-0.14)$, only postnatal smoker $(-0.12)$ and always smoker $(-0.30) \mathrm{p}=0.007$.

Conclusion We concluded that maternal smoking has an important effect on adolescence height.

\section{P2-176 ANALYSIS OF THE FAMILIAR EXPENSES WITH MEDICINES FOR DIABETES TREATMENT IN THE BRAZILIAN POPULATION}

doi:10.1136/jech.2011.142976j.11

\section{A Zaccolo, ${ }^{*}$ S Mengue. UFRGS, Porto Alegre, Rio Grande do Sul, Brazil}

Introduction Direct costs for diabetes care accounts for $2.5 \%$ to $15 \%$ of national health expenditures around the world, fee that varies according to local prevalence of diabetes and to the complexity of treatment available. Economic aspects of diabetes have been studied in the United States and in countries of Europe, but such information are still scarce in Brazil. The main objective is to evaluate the individual spending with prescription drugs to treat diabetes based 
on data contained in the Survey on Household Budgets (POF 2002-2003).

Materials and Methods This analysis is conducted with the data from POF 2002-2003 which involved interviews on a sample of 48470 families.

Results The sum of all drug costs for diabetes treatment equals US\$ 249 million. Among those who have acquired any drugs for diabetes care the average expense was US\$ 6.30. 31.1\% of the drugs for diabetes were obtained through the NHS and $64.2 \%$ were bought in a drugstore or pharmacy. Those with family incomes of up to US\$ 137.00 spend the equivalent of $4.28 \%$ the income on medicines for diabetes. While those with family incomes above US\$2000.00 have a drug spending for diabetes equivalent to $0.51 \%$ of their income.

Conclusion Brazil spends the equivalent of $0.02 \%$ of its GDP on medicines for the treatment of diabetes. The average monthly spend on medicines for diabetes is US\$ 6.30. Despite the health system in Brazil claim to be universal, only $31 \%$ of medications for diabetes were covered by the system.

\section{P2-177 ACCUMULATION OF OBESITY-RELATED EATING BEHAVIOURS AND THE INCIDENCE OF TYPE 2 DIABETES AMONG MIDDLE-AGED JAPANESE MEN AND WOMEN: THE CIRCULATORY RISK IN COMMUNITIES STUDY (CIRCS)}

doi:10.1136/jech.2011.142976j.12

\begin{abstract}
${ }^{1,2} \mathrm{~K}$ Maruyama, ${ }^{* 2} \mathrm{M}$ Kiyama, ${ }^{2,3} \mathrm{~S}$ Sato, ${ }^{1,2} \mathrm{~T}$ Ohira, ${ }^{2} \mathrm{~K}$ Maeda, ${ }^{1,2} \mathrm{H}$ Noda, ${ }^{2} \mathrm{~S}$ Nishimura, ${ }^{2} \mathrm{~A}$ Kitamura, ${ }^{2} \mathrm{~T}$ Okada, ${ }^{1,2} \mathrm{H}$ Imano, ${ }^{2} \mathrm{M}$ Nakamura, ${ }^{2} \mathrm{Y}$ Ishikawa, ${ }^{4} \mathrm{M}$ Kurokawa, ${ }^{5} \mathrm{~K}$ Yamagishi, ${ }^{1} \mathrm{H}$ Iso. ${ }^{1}$ Osaka University Graduate School of Medicine, Osaka, Japan; ${ }^{2}$ Osaka Medical Center for Health Science and Promotion, Osaka, Japan; ${ }^{3}$ Chiba Prefectural Institute of Public Health, Chiba, Japan; ${ }^{4}$ Osaka Prefecture University School of comprehensive rehabilitation, Osaka, Japan; ${ }^{5}$ University of Tsukuba Graduate School of Comprehensive Human Sciences, and Institute of Community Medicine, Tsukuba, Japan
\end{abstract}

Introduction To examine whether the accumulation of obesityrelated eating behaviours such as eating quickly, eating until full, eating right before bedtime and breakfast skipping are associated with incidence of type 2 diabetes mellitus (T2DM) among middleaged Japanese men and women.

Methods The 2840 subjects ( 890 men and 1950 women) who were aged 40-69 year and free of T2DM were enrolled at baseline survey from 2003 to 2006 in a community-based longitudinal study, the CIRCS. The subjects were asked following four eating behaviours by questionnaire at baseline: "speed of eating", "eating until full", "eating right before bedtime" and "breakfast skipping". T2DM was defined as fasting serum glucose $\geq 7.0 \mathrm{mmol} / \mathrm{l}$, non-fasting serum glucose $\geq 11.1 \mathrm{mmol} / 1$ or medication for T2DM. We followed up to March 2010, and calculated HR and 95\% coefficient interval (95\% CI) of T2DM by using Cox proportional hazard model after adjustment for potential risk factors.

Results During 7-year follow-up period, 155 cases (70 men and 85 women) had an onset of T2DM. We calculated multivariate HR (95\% CI) of T2DM for each number of eating behaviours among both men and women. Compared with persons without any eating behaviours, the multivariable HR (95\% CI) of persons with 1, 2 and $\geq 3$ of eating behaviours were 2.35 (1.06 to 5.19), 1.54 (0.65 to 3.63) and 3.24 (1.29 to 8.13), p for trend $=0.09$ in men and 0.92 (0.52 to 1.62), 0.67 (0.35 to 1.30 ) and 1.97 (0.92 to 4.22 ), $\mathrm{p}$ for trend $=0.67$ in women.

Conclusion Our finding suggests that the accumulation of obesityrelated eating behaviours raise the risk of T2DM for middle-aged Japanese men.

\section{P2-178 THE ASSOCIATIONS OF DIETARY FOLATE, VITAMIN $B_{6}$ AND $B_{12}$ INTAKES WITH RISK OF SUDDEN CARDIAC DEATH: THE CIRCULATORY RISK IN COMMUNITIES STUDY (CIRCS)}

doi:10.1136/jech.2011.142976j.13

${ }^{1,2} \mathrm{M}$ Maruyama, ${ }^{*}{ }^{1,2} \mathrm{~T}$ Ohira, ${ }^{1,2} \mathrm{H}$ Imano, ${ }^{2} \mathrm{~A}$ kitamura, ${ }^{2} \mathrm{M}$ Kiyama, ${ }^{2} \mathrm{~T}$ Okada, ${ }^{2} \mathrm{~K}$ Maeda, ${ }^{3} \mathrm{~K}$ Yamagishi, ${ }^{2,4} \mathrm{H}$ Noda, ${ }^{2} \mathrm{Y}$ Ishikawa, ${ }^{2} \mathrm{~T}$ Shimamoto, ${ }^{1} \mathrm{H}$ Iso. ${ }^{1}$ Department of Social and Environmental Medicine, Osaka University, Suita, Japan; ${ }^{2}$ Osaka Medical Center for Health Science and Promotion, Osaka, Japan; ${ }^{3}$ Department of Public Health Medicine, University of Tsukuba, Tsukuba, Japan; ${ }^{4}$ Department of Health Care Economics and Industrial Policy, Suita, Japan

Introduction To examine whether dietary folate, vitamin $\mathrm{B}_{6}$ and $\mathrm{B}_{12}$ intakes are associated with the risk of sudden cardiac death (SCD) among Japanese men and women.

Method We conducted a nested case-control study. For each case of SCD entered between 1973 and 2001 in the CIRCS, two controls were randomly selected for each case and matched for age ( \pm 3 years), sex and community from among participants without history of SCD. The 77 cases and 154 controls aged 30-84 years were enrolled. Dietary folate, vitamin $\mathrm{B}_{6}$ and $\mathrm{B}_{12}$ intakes were assessed by 1 day $24 \mathrm{~h}$ dietary recall. We calculated conditional OR and $95 \% \mathrm{CI}$ of each nutrients for risk of SCD adjusted for potential confounding factors.

Result Higher intake of folate was associated with lower risk of SCD. The multivariable adjusted OR $(95 \% \mathrm{CI})$ of highest quartile was 0.32 (95\% CI 0.12 to 0.82$)$, p for trend $=0.06$ compared with lowest quartile. However, vitamin $\mathrm{B}_{6}$ and $\mathrm{B}_{12}$ intakes were not associated with risk of SCD. The multivariable adjusted OR $(95 \%$ $\mathrm{CI})$ of highest quartile of vitamin $\mathrm{B}_{6}$ and $\mathrm{B}_{12}$ intakes were $1.25(95 \%$ CI 0.49 to 3.21 ), $p$ for trend $=0.64$ and 0.83 (95\% CI 0.34 to 1.98$), p$ for trend $=0.86$ compared with lowest quartiles, respectively.

Conclusion Our findings suggest that higher dietary folate intake is associated with lower risk of SCD among Japanese men and women

\section{P2-179 A META-ANALYSIS OF THE RELATIONSHIP BETWEEN AGE AT PUBERTY AND TESTICULAR CANCER}

doi:10.1136/jech.2011.142976j.14

${ }^{1} \mathrm{M}$ Maule, ${ }^{* 1,2} \mathrm{~J}$ A L Malavassi, ${ }^{1} \mathrm{~L}$ Richiardi. ${ }^{1}$ Cancer Epidemiology Unit, University of Turin, Torino, Italy; ${ }^{2}$ CR-Fundacion, Universidad Autonoma de Centro America, San José, Costa Rica

Introduction Testicular cancer is one of the most rapidly increasing tumour types but its aetiology is still largely unexplained. Cryptorchidism and familial testicular cancer, the most established risk factors, may explain $<10 \%$ of all cases. Among investigated postnatal factors, early puberty was suggested as a potential risk factor but the topic has been poorly investigated.

Methods We undertook a meta-analysis of the association between age at puberty and testicular cancer risk. Search strategies were conducted in PubMed on December 2010. All markers of puberty onset (age at voice change, age at first nocturnal emission, age when started shaving, and reported age at onset) were considered. We recategorised age at puberty from all the studies into a common threelevel variable (younger age than peers, same age as peers, older age then peers), while analyses using quantitative information are ongoing.

Results A total of 348 references were retrieved, of which 11 met the inclusion criteria. Later puberty appeared to be protective. In particular later vs same age at reported onset of puberty gave an $\mathrm{OR}$ of 0.65 (95\% CI 0.52 to 0.78 ) based on three studies, later vs same age at voice change an OR of 0.79 (95\% CI 0.68 to 0.92 ) based on five studies, and later vs same age at start shaving an OR of 0.84 (95\% CI 0.75 to 0.95 ) based on five studies. Early puberty showed a smaller or no protective effect. 